\title{
Особенности МДП-структур на основе изолирующих пленок PbSnTe:In с составом вблизи инверсии зон, обусловленные их сегнетоэлектрическими свойствами
}

\author{
(C) А.Э. Климов ${ }^{1,2}$, А.Н. Акимов ${ }^{1}$, И.О. Ахундов ${ }^{1}$, В.А. Голяшов ${ }^{1,3}$, Д.В. Горшков ${ }^{1}$, \\ Д.В. Ищенко ${ }^{1}$, Е.В. Матюшенко ${ }^{1}$, И.Г. Неизвестный ${ }^{1,2}$, Г.Ю. Сидоров ${ }^{1}$, \\ С.П. Супрун ${ }^{1}$, А.С. Тарасов ${ }^{1}$, О.Е. Терещенко ${ }^{1,3}$, В.С. Эпов ${ }^{1}$
}

${ }^{1}$ Институт ффизики полупроводников им. А.В. Ржанова Сибирского отделения Российской академии наук, 630090 Новосибирск, Россия

${ }^{2}$ Новосибирский государственный технический университет, 630073 Новосибирск, Россия

${ }^{3}$ Новосибирский государственный университет, 630090 Новосибирск, Россия

E-mail: klimov@isp.nsc.ru

Поступила в Редакцию 15 апреля 2020 г.

В окончательной редакции 21 апреля 2020 г.

Принята к публикации 21 апреля 2020 г.

Исследованы характеристики МДП-структур на основе изолирующих пленок PbSnTe:In с составами вблизи инверсии зон, полученных методом молекулярно-лучевой эпитаксии. Показано, что ряд их особенностей могут быть вызваны сегнетоэлектрическим фазовым переходом с температурой Кюри в области примерно $T \approx 15-20 \mathrm{~K}$

Ключевые слова: твердый раствор PbSnTe:In, эффект поля, МДП-структура, сегнетоэлектрический фазовый переход.

DOI: $10.21883 /$ FTP.2020.10.49955.29

\section{1. Введение}

Ширина запрещенной зоны твердого раствора $\mathrm{Pb}_{1-x} \mathrm{Sn}_{x}$ Те зависит от состава и температуры и лежит в интервале $E_{g} \approx 0-0.4$ эВ. В настоящее время особый интерес связан с составами вблизи $x_{\text {inv }} \approx 0.35$, где при гелиевых температурах происходит инверсия краев разрешенных зон, а $E_{g}$ стремится к нулю. В области инверсии зон $x>x_{\text {inv }}$ PbSnTe находится в фазе топологического кристаллического изолятора (ТКИ) [1], а в области $x \approx 0.22-0.3<x_{\text {inv }}$ при добавлении в $\mathrm{PbSnTe}$ индия с концентрацией до нескольких ат\% может быть реализовано „изолирующее“ состояние $\mathrm{PbSnTe}: \mathrm{In}$ с крайне низкой проводимостью [2]. Имеются данные, что ТКИ-фаза может наблюдаться в пленках PbSnTe:In даже при $x<0.35$ [3], а „изолирующее“ состояние в том числе и при $x>0.3$ [4]. Таким образом, имеется потенциальная возможность совместить эти две особенности $\mathrm{PbSnTe}$ в одной области $x \approx 0.3-0.35$. Это представляет особый интерес для задач спинтроники, так как в этом случае связанная с дираковскими состояниями поверхностная проводимость не будет „шунтироваться“ высокой проводимостью объема.

О возможности сегнетоэлектрического фазового перехода (СЭФП) в PbSnTe определенного состава в литературе упоминается достаточно давно. Так, в $[5,6]$ фазовый переход в $\mathrm{SnTe}$ вблизи $T=75-77 \mathrm{~K}$ проявляется в виде выраженной особенности на температурных зависимостях коэффициента расширения. Сделано предположе- ние, что при этом SnTe переходит из ромбоэдрической модификации в высокотемпературную кубическую. В работе [7] методом магнитоплазменного отражения исследован $\mathrm{Pb}_{1-x} \mathrm{Sn}_{x}$ Те в области составов $x=0-0.4$ в диапазоне температур $T=4.2-100 \mathrm{~K}$. Указывается, что постоянная Кюри оказалась практически одинаковой для всех исследованных составов, а для $x=0.4$ СЭФП наблюдается при температуре Кюри $T_{\mathrm{C}}=20 \mathrm{~K}$. В работе [8] приводятся данные по зависимости $T_{\mathrm{C}}$ от состава в диапазоне $x=0-1$. Для образцов, полученных разными методами, такие зависимости несколько отличаются. Переход от нормального к виртуальному сегнетоэлектрику с отрицательной $T_{\mathrm{C}}$ наблюдается примерно при $x<0.2-0.3$. В работе [9] с учетом межзонной гибридизации рассчитана концентрационная зависимость $T_{\mathrm{C}}$ для разных составов. Отмечается, что условия для структурного фазового перехода более благоприятны в инверсионной области составов с $x>0.35$. В работе [10] отмечается возможность существования в PbSnTe нескольких фазовых переходов, а в [11] обобщен ряд экспериментальных данных по зависимости $T_{\mathrm{C}}$ от состава, полученных разными авторами на образцах, изготовленных разными методами. Согласно этой работе, $T_{\mathrm{C}} \approx-(65-50) \mathrm{K}$ для $\mathrm{PbTe}$, а переход к положительной $T_{\mathrm{C}}$ происходит в области $x \approx 0.2-0.3$. Одновременно в эту же область составов попадает и несколько значений $T_{\mathrm{C}}>0$, вплоть до $T_{\mathrm{C}} \approx 30 \mathrm{~K}$. В работе [12] исследованы пленки $\mathrm{Pb}_{0.75} \mathrm{Sn}_{0.25} \mathrm{Te}:$ In с концентрацией индия $\sim 0.5 \%$ и указывается на существование 
СЭФП с $T_{\mathrm{C}} \approx 18 \mathrm{~K}$. В работе [13] в температурном диапазоне $T=4.2-40 \mathrm{~K}$ детально исследованы сегнетоэлектрические свойства объемных монокристаллов $\mathrm{Pb}_{0.75} \mathrm{Sn}_{0.25} \mathrm{Te}: \mathrm{In}$ с содержанием индия 0.1, 0.5 и $1.0 \%$ и подвижностью электронов $(T=30 \mathrm{~K}) 1.5 \cdot 10^{4}, 4 \cdot 10^{5}$ и $4.5 \cdot 10^{5} \mathrm{~cm}^{2} \cdot \mathrm{B}^{-1} \cdot \mathrm{c}^{-1}$ соответственно. Измерялись температурные зависимости спонтанного напряжения и низкочастотной диэлектрической проницаемости $\varepsilon$, поляризация, а также вольт-амперные характеристики в режиме токов, ограниченных пространственным зарядом (ТОПЗ). Для образцов с содержанием индия 1.0 и $0.5 \%$ найдено $T_{\mathrm{C}}=21$ и $22.5 \mathrm{~K}$, а в области $T \approx 4.2-10 \mathrm{~K}$ для этих же концентраций индия $\varepsilon \approx 3300$ и $\varepsilon \approx 5000$ соответственно. Измерения ряда сегнетоэлектрических параметров удалось выполнить благодаря низкой проводимости исследованных образцов $\mathrm{Pb}_{0.75} \mathrm{Sn}_{0.25} \mathrm{Te}: \mathrm{In}$ в области $T<T_{\mathrm{C}}$. Как в работе [12], так и в [13] подчеркивается сильное влияние примеси индия на сегнетоэлектрические свойства PbSnTe, которое выражается, в том числе, в существенном изменении величины $T_{\mathrm{C}}$. Авторами [14] проанализированы и обобщены некоторые особенности низкотемпературных фазовых переходов в твердых четверных растворах соединений $\mathrm{A}^{\mathrm{IV}} \mathrm{B}^{\mathrm{VI}}$, обусловленных, в том числе, атомами примеси. В [15] резкое (до 100 раз) увеличение диэлектрической проницаемости при увеличении температуры от $T \approx 10$ до $T \approx 25 \mathrm{~K}$ наблюдалось на образцах $\mathrm{Pb}_{0.74} \mathrm{Sn}_{0.26} \mathrm{Te}: \mathrm{In}$ (содержание индия $\sim 3 \%$ ), полученных методом молекулярно-лучевой эпитаксии (МЛЭ) на подложках (111) $\mathrm{BaF}_{2}$. В работе [16] на аналогичных образцах с $x \approx 0.29$ вблизи $T \approx 22 \mathrm{~K}$ обнаружена ярко выраженная особенность на температурных зависимостях постоянных времени фотопроводимости. Делается предположение, что она может быть связана также с СЭФП.

Таким образом, на основе известных данных можно сказать следующее: РbТе является виртуальным, а $\mathrm{SnTe}$ - нормальным сегнетоэлектриками. Существование СЭФП в PbSnTe и PbSnTe:In определенного состава не вызывает сомнений, а область перехода от нормального к виртуальному сегнетоэлектрику (с отрицательной $T_{\mathrm{C}}$ ) приходится на $x \approx 0.2-0.3$. Наблюдается сильная зависимость сегнетоэлектрических свойств PbSnTe от характера и концентрации примесей, к которым можно отнести, в том числе, электрически активные собственные точечные дефекты, а также индий в PbSnTe:In. Вследствие этого имеется довольно большой разброс значений $T_{\mathrm{C}}$ для составов вблизи интервала $x \approx 0.2-0.3$, в котором проводимость PbSnTe:In может быть крайне низка. Чаще для этой области $x$ упоминаются значения $T_{\mathrm{C}} \approx 20 \mathrm{~K}$ и, во всяком случае, $T_{\mathrm{C}}>0$ при $x>0.24-0.26$. Следовательно, составы исследованных в настоящей работе пленок PbSnTe:In таковы, что при гелиевых температурах они должны находиться в сегнетоэлетрической фазе. При этом спонтанная и вынужденная поляризация, а также доменная структура пленок могут проявляться в ряде особенностей PbSnTe:In МДП-структур и, по-видимому, должны учи- тываться в дальнейшем при анализе свойств, связанных с ТКИ-особенностями PbSnTe.

\section{2. Образцы и методики измерений}

Экспериментальные структуры были изготовлены на основе монокристаллической пленки $\mathrm{PbSnTe}: \mathrm{In} /(111) \mathrm{BaF}_{2}$ толщиной 1.7 мкм, полученной методом МЛЭ. Состав пленки $x \approx 0.28$, содержание индия $\sim 0.7 \mathrm{aT} \%$, подвижность электронов вблизи $T \approx 40 \mathrm{~K}$ составила $\sim 3.6 \cdot 10^{4} \mathrm{~cm}^{2} \cdot \mathrm{B}^{-1} \cdot \mathrm{c}^{-1}$. В качестве диэлектрика в МДП-структурах использовалась 8-микронная майларовая пленка. В отдельных экспериментах на поверхность $\mathrm{PbSnTe}$ после химической обработки методом атомно-слоевого осаждения наносился слой $\mathrm{Al}_{2} \mathrm{O}_{3}$ толщиной 72 нм. Схематичное изображение экспериментальной структуры приведено на рис. 1. Исток и сток $n^{+}$-типа проводимости были разделены „изолирующим“ каналом шириной 50 мкм и длиной $\sim 0.1 \mathrm{~cm}$. Области истока и стока были сформированы вакуумным напылением индия толщиной $\sim 100$ нм с последующим диффузионным отжигом. Как и в исходной пленке, концентрация и подвижность электронов в этих областях контролировались с использованием эффекта Холла и составили $n \approx 3 \cdot 10^{17} \mathrm{~cm}^{-3}, \mu_{n}>1000 \mathrm{~cm}^{2} \cdot \mathrm{B}^{-1} \cdot \mathrm{c}^{-1}$ во всем исследованном температурном интервале. Измерялись зависимости тока в канале $I_{s d}$ от напряжения исток/сток $U_{s d}$ при различном напряжении на затворе $U_{\text {gate, }}$ а также временны́е зависимости $I_{s d}(t)$ при фиксированных значениях $U_{s d}$ и равномерном изменении $U_{\text {gate }}$ с различной скоростью $U_{\text {gate }}^{\prime}$. Диапазоны затворного напряжения и скорости его изменения составили $-1250<U_{\text {gate }}<+1250$ В и $U_{\text {gate }}^{\prime}=2.5-250$ В $/$ с соответственно. Минимальная величина измерявшегося тока (последний разряд использованного цифрового амперметра) составила $10^{-11}$ А. Измерения проводились непосредственно в жидком гелии или в его парах в экранированной от фонового излучения металлической камере.

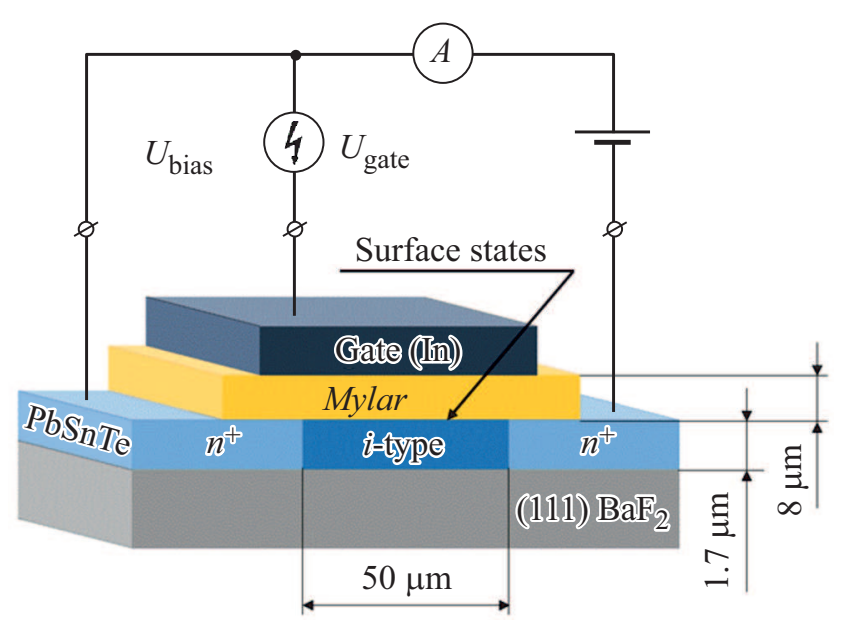

Рис. 1. Схематичное изображение экспериментальной структуры. 


\section{3. Экспериментальные результаты}

На рис. 2 в двойном логарифмическом масштабе приведены вольт-амперные характеристики (BAX) канала МДП-структуры, измеренные при $T=4.2 \mathrm{~K}$ при нулевом, а также при максимальных отрицательном и положительном значениях $U_{\text {gate }}$. Время развертки $U_{s d}$ от нуля до максимального значения составило в данном случае $t_{\mathrm{CVC}} \approx 700$ с. Каждая ВАХ измерялась после охлаждения образца от комнатной температуры до $T=4.2 \mathrm{~K}$ примерно за одинаковое время. В этом случае ВАХ, измеренные в одинаковых условиях, воспроизводились. $\mathrm{BAX}$, измеренные повторно в одинаковых условиях, но без отогревания образцов до $T=300 \mathrm{~K}$, существенно отличаются. Это связано с наличием в образцах глубоких ловушек с большими постоянными времени, заряд которых меняется при измерении BAX и не возвращается к исходному значению при $T=4.2 \mathrm{~K}$ в течение длительного времени (до суток и более) после окончания измерений. Качественно поведение ВАХ соответствует модели токов, ограниченных пространственным зарядом (ТОПЗ) при наличии ловушек. Так, для этого механизма протекания тока характерна зависимость $I \propto U^{2}$ в области больших напряжений (полное заполнение ловушек) и малых напряжений (ловушки заполнены не полно-

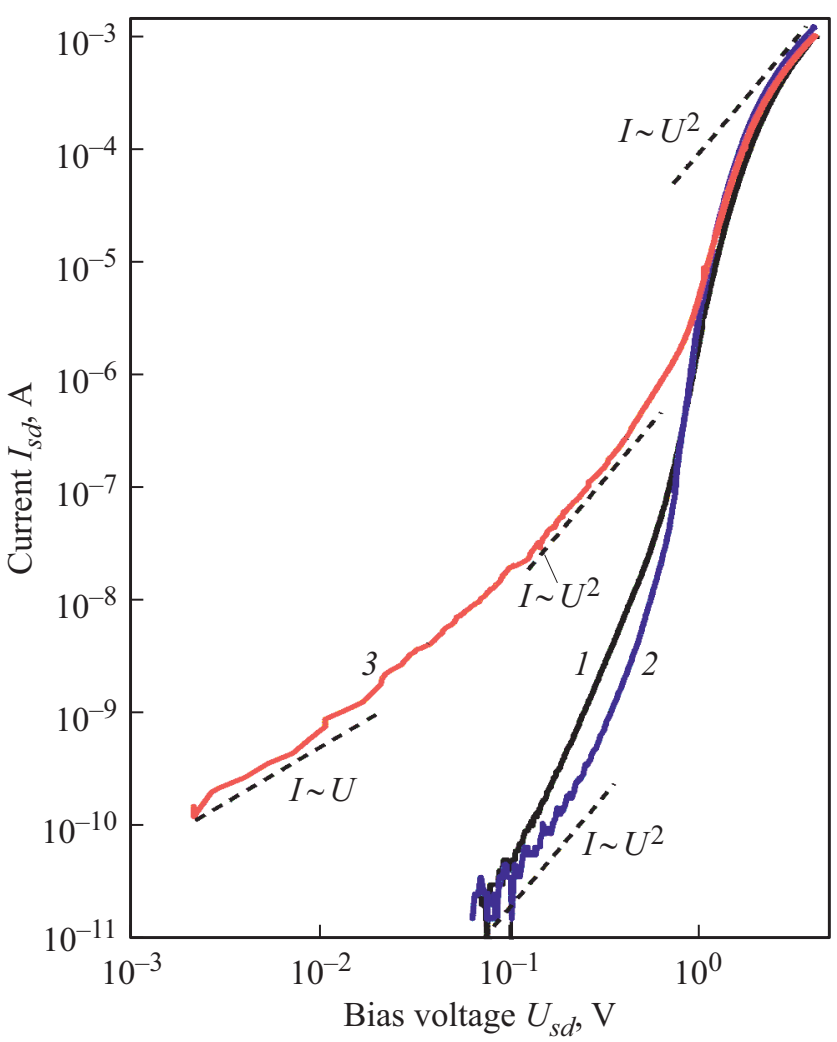

Рис. 2. Вольт-амперные характеристики PbSnTe:In МДПструктуры. Температура измерений $T=4.2 \mathrm{~K}$. Затворное напряжение $U_{\text {gate }}$ B: $1-0,2--1250,3-+1250$. Пунктирные отрезки соответствуют линейной и квадратичной зависимостям тока от напряжения. стью). Поведение BAX в промежуточной области напряжений может быть сложным при наличии различных типов ловушек или при их непрерывном энергетическом спектре [17]. Из рисунка видно, что при $U_{\text {gate }}=+1250 \mathrm{~B}$ наблюдается сильное (до $10^{3}$ раз) увеличение $I_{s d}$ в области малых $U_{s d}$. Необходимо отметить, что из-за наличия медленных ловушек соотношения между ВАХ, измеренными при различных значениях $U_{\text {gate }}$, существенно отличаются для разных $t_{\mathrm{CVC}}$ (скоростей изменения $\left.U_{s d}\right)$. Однако результаты и их обсуждение, связанные с переходными процессами, обусловленными сложным спектром ловушек, выходят за рамки настоящей работы. Далее будут приведены некоторые данные, полученные при измерении $I_{s d}$ в области небольших значений $U_{s d}$ и положительном $U_{\text {gate }}$ (наибольшее изменение $I_{s d}$ под действием затворного напряжения) и полученные в области больших значений $U_{s d}$.

На рис. 3 приведена серия временны́х зависимостей $I_{s d}(t)$, измеренных при разных температурах при $U_{s d}=0.143 \mathrm{~B}$. Вблизи этого напряжения наблюдалось наибольшее изменение $I_{s d}$ под действием затворного напряжения. Сплошные кривые получены без дополнительной обработки поверхности $\mathrm{PbSnTe}: \mathrm{In}$, пунктирная $(T=4.2 \mathrm{~K})$ - после обработки поверхности в насыщенном парами $\mathrm{HCl}$ изопропиловом спирте [18], нанесении слоя $\mathrm{Al}_{2} \mathrm{O}_{3}$ и выдержке образца в атмосфере в течение 32 сут.

Из рис. $3, a$ видно, что вплоть до $T=12.2 \mathrm{~K}$ в интервале $t=0-50 \mathrm{c}$, где $U_{\text {gate }}$ растет линейно со временем, ток также возрастает. На этом участке его увеличение может достигать $\sim 10^{6}$ раз (пунктирная кривая). Далее, от $t=50$ с и вплоть до моментов, помеченных стрелками, $U_{\text {gate }}=+1250 \mathrm{~B}$, а ток неэкспоненциально уменьшается. Затем $U_{\text {gate }}$ начинает уменьшаться с той же скоростью, с какой увеличивалось при $t<50$ с. При этом ток сначала резко падает за время $\Delta t<50 \mathrm{c}$, а затем на зависимостях наблюдаются переходные процессы, продолжающиеся и тогда, когда $U_{\text {gate }}=0$. Характер этих процессов, как и форма кривых $I_{s d}(t)$ на других участках, существенно трансформируются с ростом температуры. Радикальное изменение поведения $I_{s d}(t)$ происходит начиная с $T=14.6 \mathrm{~K}$. В увеличенном масштабе это показано на рис. $3, b$. Видно, что при $T=14.6$ и $18.8 \mathrm{~K}$ относительное изменение $I_{s d}(t)$ под действием $U_{\text {gate }}$ существенно ниже, чем при меньших температурах. Для $T=24.4 \mathrm{~K}$ это изменение настолько мало, что в выбранном масштабе рисунка не видно. Также видна существенная трансформация формы зависимостей $I_{s d}(t)$ при этих температурах. Так, при $T=18.8 \mathrm{~K}$ в области постоянного $U_{\text {gate }}=+1250$ В наблюдаются автоколебания тока.

Из-за сложного и качественно различающегося поведения $I_{s d}(t)$ при разных температурах, для его описания в качестве численного параметра нами было выбрано максимальное относительное изменение тока $\Delta I_{s d}(t) / I_{s d}(0)$, вызванное приложением $U_{\text {gate }}$. Его зависимость от температуры приведена на рис. 4 вместе с температурной зависимостью тока при $U_{\text {gate }}=0$. Из 
рисунка видно, что в интервале $T=4.2-25 \mathrm{~K}$ величина $\Delta I_{s d}(t) / I_{s d}(0)$ падает на $\sim 8$ порядков, при этом в области $T \approx 15-20 \mathrm{~K}$ наблюдается выраженная особенность в виде „ступеньки“.

На рис. 5 приведены затворные характеристики МДПструктуры при $T=4.2 \mathrm{~K}$ до дополнительной обработки
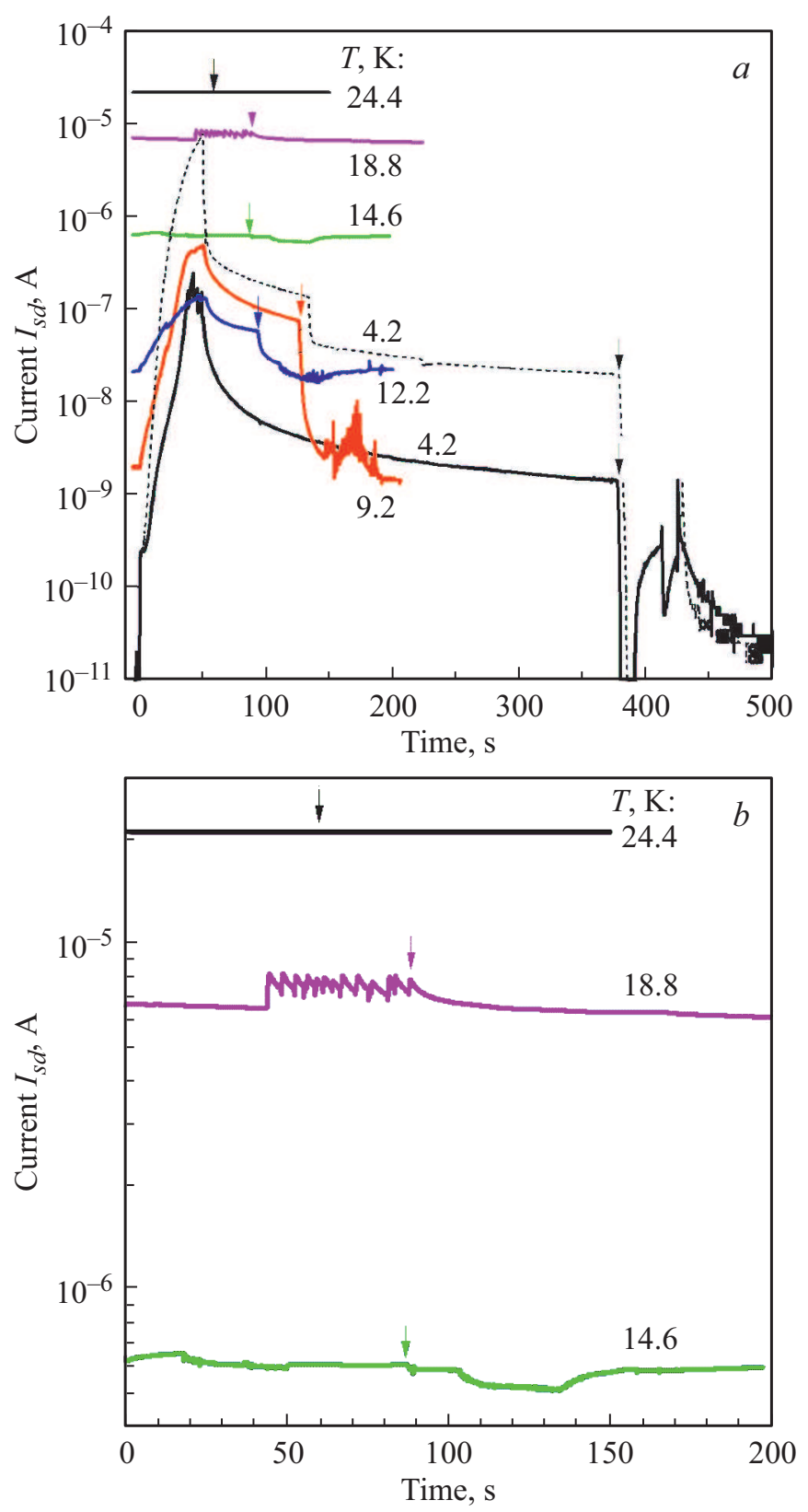

Рис. 3. Временнб́е зависимости тока $I_{s d}(t)$ при разных температурах $(a)$ и для трех значений температуры в увеличенном масштабе $(b)$. Для всех кривых в интервале $t=0-50 \mathrm{c}$ $U_{\text {gate }}$ линейно увеличивалось от нуля до $U_{\text {gate }}=+1250 \mathrm{~B}$ $\left(U_{\text {gate }}=25 \mathrm{~B} / \mathrm{c}\right)$, затем оставалось постоянным до моментов времени, помеченных стрелками вблизи соответствующих кривых, после чего также за 50 с линейно уменьшалось до нуля. $U_{s d}=0.143$ В. Температура измерений для каждой кривой приведена на рисунке. Пунктирная кривая получена после дополнительной обработки поверхности (см. текст).

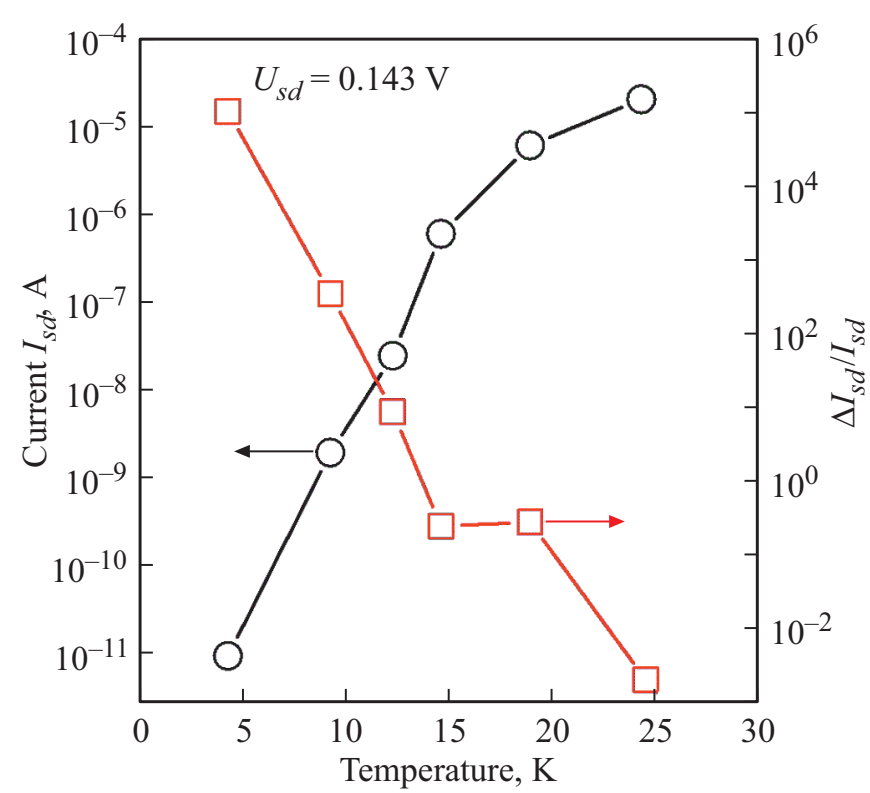

Pис. 4. Температурные зависимости тока исток/сток $I_{s d}$ при $U_{\text {gate }}=0$ (круги, левая шкала) и максимального относительного изменения $\Delta I_{s d}(t) / I_{s d}(0)$ при приложении затворного напряжения $U_{\text {gate }}=+1250$ В (квадраты, правая шкала).

поверхности для двух значений $U_{s d}$ в области больших напряжений. Выраженный гистерезис на характеристиках обусловлен „медленными“ локализованными состояниями. Видно, что в областях $U_{\text {gate }}$ примерно от -0.4 до $+1.25 \mathrm{\kappa B}$ (верхние ветки) и от -1.25 до $+0.4 \mathrm{\kappa B}$ (нижние ветки) видны характерные повторяющиеся „выбросы“", отсутствующие при уменьшении абсолютного значения затворного напряжения от $\left|U_{\text {gate }}\right|=1.25 \mathrm{\kappa B}$ примерно до $\left|U_{\text {gate }}\right| \approx 0.4$ кВ.

\section{4. Обсуждение результатов}

Из рис. 2 следует, что при $U_{\text {gate }}=0$ ток в канале $\mathrm{PbSnTe}: \mathrm{In}$ МДП-структуры определяется токами, ограниченными пространственным зарядом. В режиме ТОПЗ в области между истоком и стоком формируется пространственный заряд с нарушением электронейтральности объема. В нашем случае вследствие $n^{+}$-типа проводимости истока и стока пространственный заряд формируют инжектируемые из контакта электроны. В стационарном режиме инжектированные в канал МДП-структуры электроны определенным образом распределены между уровнями захвата (ловушками) и зоной проводимости в соответствии со статистикой Ферми-Дирака. Распределение электрического поля $E$ и полного (свободного и локализованного) заряда задается уравнением Пуассона. В общем случае плотность инжектированного заряда падает, а напряженность $E$ растет от истока к стоку. Расчет их пространственных распределений в аналитическом виде для произвольного случая невозможен. Это связано, в частности, с тем, что на 
разных расстояниях от контактов определяющие ТОПЗ ловушки могут быть заполнены как полностью, так и не полностью. Геометрия исследованной структуры такова, что межконтактное расстояние более чем на порядок превышает толщину пленки. В этом случае нарушение электронейтральности инжектированным зарядом ведет к появлению поперечной компоненты Е и соответствующему распределению заряда по толщине пленки, также зависящему от расстояния от контактов. Кроме того, такая геометрия образца должна вести к тому, что величину ТОПЗ, а также распределение заряда и $E$ будут определять не только ловушки в объеме канала, но и ловушки на поверхности пленки. При этом, как указывалось ранее в [17], ловушки в PbSnTe:In могут быть различных типов, вплоть до существования ловушек с непрерывным энергетическим спектром. Из сказанного следует, что пространственные распределения заряда и электрического поля, задающие величину тока для каждого значения $U_{s d}$, определяются многими факторами и могут быть очень сложными. Поэтому появление некоего дополнительного фактора, оказывающего хотя бы незначительное влияние на обсуждаемые распределения, может вести к заметному изменению тока. Одним из таких дополнительных факторов может быть встроенное электрическое поле, связанное с доменной структурой образца в сегнетоэлектрической фазе, и резкое изменение этого поля при вынужденной смене направления поляризации отдельного домена. Обратимся теперь к результатам, представленным на рис. 5. Известно (см., например, [5]), что в PbSnTe вектор спонтанной поляризации $\mathbf{P}$ направлен вдоль оси [111]. Упрощенно, это означает существование, как минимум, восьми независимых направлений $\mathbf{P}$ для отдельных доменов. Допустим, что в канале МДП-структуры существует некоторое количество сегнетоэлектрических доменов, которые способны менять свою поляризацию под действием поперечного поля затвора, максимальная величина которого при $U_{\text {gate }}=1.25 \mathrm{\kappa B}$ составляет $\sim 1.5 \cdot 10^{6} \mathrm{~B} /$ см. В пленке $\mathrm{PbSnTe}$, только за счет $\varepsilon$, даже без учета экранировки поверхностным зарядом, поле затвора ослабляется в 1000 и более раз и становится сравнимым с характерной величиной тянущего поля исток/сток $(\sim 500 \mathrm{~B} / \mathrm{cm}$ для $\left.U_{s d}=2.5 \mathrm{~B}\right)$. В этом случае, если „выбрось“ на рис. 5 связаны с переполяризацией доменов, это происходит под воздействием суперпозиции поля, наведенного затвором и сравнимого по величине тянущего поля в канале, разного на разном расстоянии от контактов. C учетом разных направлений спонтанной поляризации это может привести к тому, что разные домены будут переполяризовываться в широком диапазоне напряжений затвора, как это видно на рис. 5. В рамках такой модели наличие участков кривых на рис. 5, на которых „выбросы“" отсутствуют, связано с тем, что для „обратной переполяризации“ домена электрическое поле должно сменить знак и достигнуть некой критической величины. Однако в рамках рассматриваемой модели, судя по положениям „выбросов“, это происходит еще до смены знака
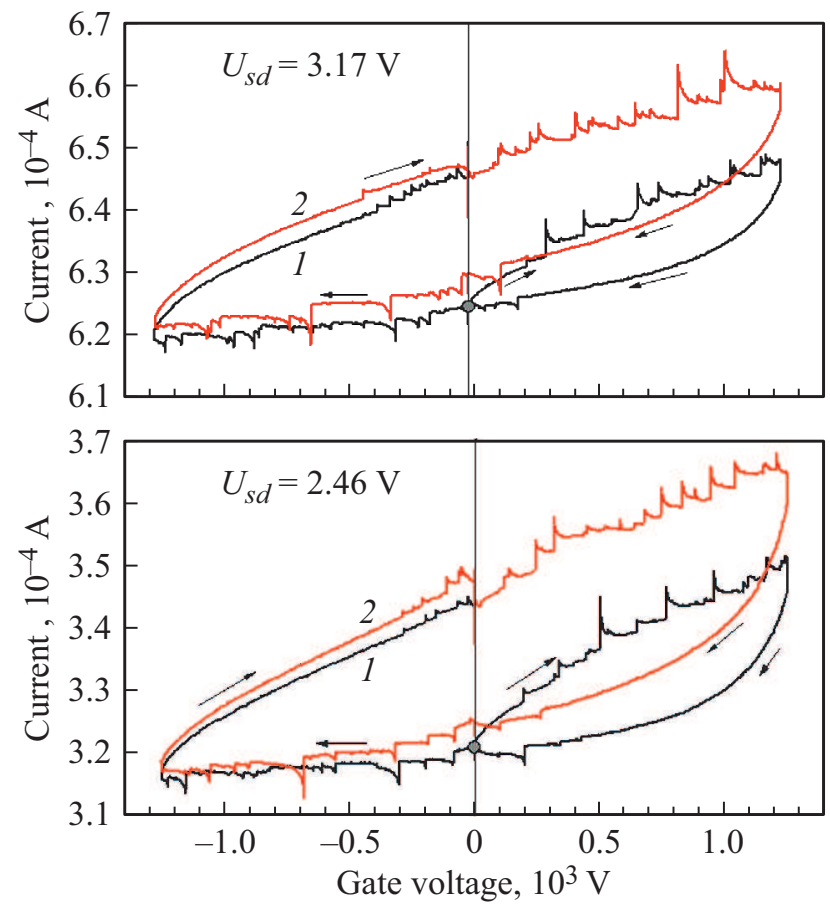

Рис. 5. Зависимости тока $I_{s d}$ от напряжения затвора $U_{\text {gate. }}$ Значения $U_{s d}$ приведены на рисунках. Температура измерений $T=4.2 \mathrm{~K}$. Скорость изменения затворного напряжения $U_{\text {gate }}^{\prime}= \pm 25 \mathrm{~B} / \mathrm{c}$. Начальные точки измерений при $U_{\text {gate }}=0$ обозначены кругами. Направления изменения $U_{\text {gate }}$ показаны стрелками вблизи соответствующих участков зависимостей. На каждом рисунке приведено по два последовательных цикла изменений $U_{\text {gate }}$ в интервале $-1250 \mathrm{~B}<U_{\text {gate }}<+1250 \mathrm{~B}$.

напряжения затвора! На качественном уровне это объясняется следующим образом. При сравнительно быстром „включении“ $U_{\text {gate }}>0$ поле затвора сначала экранируется отрицательным зарядом свободных электронов, инжектируемых из контакта. Это ведет к заметному росту тока $I_{s d}$ как на рис. 2 (кривая 3 ), так и на рис. 3 на соответствующем участке $t<50 \mathrm{c}$. Одновременно идет захват свободных электронов на ловушки, что ведет к последующему снижению тока на рис. 3. Как видно из рис. 3 , а также, судя по гистерезисным петлям на рис. 5, характерные времена таких процессов (времена захвата заряда на ловушки и выброса с них) велики и могут достигать сотен секунд и более. Тот факт, что обработка поверхности существенно влияет на характеристики МДП-структур (рис. $3, T=4.2 \mathrm{~K}$ ), говорит о том, что значительная часть таких ловушек расположена на поверхности PbSnTe или вблизи нее. Как следствие, быстрое уменьшение $U_{\text {gate }}$ от его максимального значения приведет к тому, что сформированный на поверхности ,долгоживущий“ отрицательный заряд электронов создаст отрицательное по отношению к объему пленки поле, сравнимое по величине с максимальным полем затвора. С этим и связано то, что на рис. 5 при $U_{\text {gate }}>0$ на нижних ветках „выбрось“" начинают появляться при- 
мерно при $U_{\text {gate }}=0.4 \mathrm{\kappa B}$. Хотя поле затвора при этом еще положительно, поле „наведенного“ отрицательного заряда уже его превышает. При $U_{\text {gate }}<0$ ситуация аналогична с той лишь разницей, что „наведенный“ затворным напряжением поверхностный заряд в этой области положительный. Он может формироваться, например, вследствие частичного опустошения тех же поверхностных ловушек, которые при $U_{\text {gate }}>0$ захватывают электроны. Как следует из рис. 2, появление такого заряда не сопровождается сильным изменением проводимости канала, т.е. роль свободных дырок в экранировке поля затвора в этом случае невелика. Это связано с низкой концентрацией равновесных дырок и незначительной по сравнению с темпом захвата и выброса на ловушки тепловой межзонной генерацией в объеме пленки.

В рамках рассматриваемого подхода основные особенности кривых на рис. 3 могут быть описаны на качественном уровне следующим образом. При низких температурах в области $t<50$ с быстрое нарастание $U_{\text {gate }}$ сопровождается инжекцией свободных электронов из истока в канал МДП-структуры. Свободные электроны экранируют значительную часть $U_{\text {gate }}$ с увеличением тока $I_{s d}$. Далее при постоянном $U_{\text {gate }}=+1250 \mathrm{~B}$ свободные электроны захватываются ловушками на поверхности пленки с уменьшением $I_{s d}$, так как полный отрицательный заряд, экранирующий $U_{\text {gate }}$, остается неизменным. Как видно из рис. 3 , в этом интервале $I_{s d}$ может уменьшаться на порядок и более. Это означает, что к моментам, показанным стрелками, $U_{\text {gate }}=+1250 \mathrm{~B}$ экранируется практически полностью поверхностным зарядом, связанным с перезарядкой достаточно медленных поверхностных ловушек. Поэтому последующее быстрое уменьшение положительного $U_{\text {gate }}$ ведет к тому, что объем канала оказывается под действием отрицательного электрического поля поверхностных ловушек, что и ведет к резкому уменьшению тока за счет снижения концентрации свободных электронов в канале. Последующие более длительные переходные процессы сложного характера связаны, по- видимому, со сложной динамикой возвращения поверхностных ловушек разного типа (и с разными постоянными времени) в равновесное состояние при уменьшении $U_{\text {gate. }}$ В рамках такого подхода изменение с ростом температуры формы кривых на рис. 3 может быть связано, в том числе, с изменением параметров ловушек. Следует отметить, что если предположение о роли доменной структуры в наблюдаемых эффектах верно, то вблизи температуры СЭФП можно ожидать особенно заметные изменения характеристик.

Возможные неоднородности состава PbSnTe:In, на которые указывается, например, в [19], могут вести еще и к тому, что разные домены будут иметь отличающиеся значения $T_{\mathrm{C}}$. В нашем случае из рис. 4 видно, что характерная температурная область, где, по-видимому, реализуется СЭФП, составляет примерно $T_{\mathrm{C}}=15-20 \mathrm{~K}$. Это хорошо коррелирует с литературными данными.
Из рассматриваемого подхода количество „выбросов“ на соответствующих участках кривых рис. 5 должно соответствовать количеству доменов, меняющих поляризацию под действием $U_{\text {gate }}$. Из рисунка видно, что их число составляет примерно 20-30. Поэтому, исходя из геометрии канала МДП-структуры, можно предположить, что характерный размер домена близок к ширине канала - около 50 мкм. Уменьшение площади канала должно вести к уменьшению числа доменов в нем и, соответственно, количества „выбросов““ на затворных характеристиках с ростом их относительной величины. Например, при легко реализуемых размерах канала порядка $10 \times 10$ мкм или несколько больше можно ожидать, что в нем будет находиться только один домен. В этом случае эффекты, связанные с его вынужденной переполяризацией, должны быть особенно сильными. Такие эксперименты готовятся в дальнейшем.

\section{5. Заключение}

Ряд особенностей характеристик МДП-структур на основе пленок PbSnTe:In находит объяснение в рамках модели, предполагающей наличие в них сегнетоэлектрического фазового перехода и „медленных“ поверхностных состояний. Это необходимо учитывать при анализе свойств PbSnTe: In с составами, близкими к фазе топологического изолятора, так как в этой же области могут проявляться и сегнетоэлектрические свойства.

\section{Финансирование работы}

Работа выполнена при частичной поддержке РФФИ (грант № 20-02-00324) и РНФ (грант № 17-12-01047).

\section{Конфликт интересов}

Авторы заявляют, что у них нет конфликта интересов.

\section{Список литературы}

[1] T. Liang, S. Kushwaha, J. Kim, Q. Gibson, J. Lin, N. Kioussis, R.J. Cava, N.P. Ong. Sci. Adv., 3, e1602510 (2017).

[2] Б.А. Волков, Л.И. Рябова, Д.Р. Хохлов. УФН, 172 (8), 875 (2002).

[3] Y. Tanaka, T. Sato, K. Nakayama, S. Souma, T. Takahashi, Z. Ren, M. Novak, K. Segawa, Y. Ando. Phys. Rev. B, 87, 155105 (2013).

[4] A.N. Akimov, D.V. Ishchenko, A.E. Klimov, I.G. Neizvestny, N.S. Paschin, V.N. Sherstyakova, V.N. Shumsky. Russ. Microelectron., 42 (2), 59 (2013).

[5] С.И. Новикова, Л.Е. Шелимова. ФТТ, 7 (8), 2544 (1965).

[6] С.И. Новикова, Л.Е. Шелимова. ФТТ, 9 (5), 1336 (1967).

[7] S. Nishi, H. Kawamura, K. Murase. Phys. Status Solidi B, 97, 581 (1980).

[8] K. Murase, S. Nishi. Phys. Narrow Gap Semicond., 152, 261 (1982).

[9] В.К. Дугаев, В.Л. Волков, В.И. Литвинов, К.Д. Товстюк. УФЖ, 24 (4), 538 (1979). 
[10] Р.А. Насыббулин, Р.Х. Калимуллин, В.В. Шапкин, Ю.С. Харионовский, А.М. Джумиго, Э.В. Бурсиан. ФТТ, 23 (1), 300 (1981).

[11] Р.А. Насыббулин, Я.Н. Гиршберг, Н.Н. Трунов, Р.Х. Калимуллин, А.А. Кухарский, Ю.С. Харионовский, В.В. Шапкин, Э.В. Бурсиан. ФТТ, 25 (4), 784 (1983).

[12] A. Martinez, R.J. Abbundi, B. Houston, J.L. Davis, R.S. Allgaier. J. Appl. Phys., 57, 1165 (1985).

[13] В.С. Виноградов, И.В. Кучеренко. ФТТ, 33 (9), 2572 (1991).

[14] A.I. Lebedev, I.A. Sluchinskaya. J. Alloys Compd., 203 (1-2), 51 (1994).

[15] A.E. Klimov, V.N. Shumsky. Optoelectron. Instrumentation and Date Processing, 3, 53 (2001).

[16] А.Н. Акимов, А.Э. Климов, И.Г. Неизвестный, В.Н. Шумский, В.С. Эпов. ФТП, 50 (4), 447 (2016).

[17] A.E. Klimov, V.N. Shumsky. Physica B, 404 (23-24), 5028 (2009).

[18] O.E. Tereshchenko, S.I. Chikichev, A.S. Terekhov. J. Vac. Sci. Technol. A, 17, 2655 (1999).

[19] Г.А. Калюжная, К.В. Киселева. Тр. Физ. ин-та им. П.Н. Лебедева, 177, 5 (1987).

Редактор Г.А. Оганесян

\section{Features of MIS structures based on insulating PbSnTe:In films in the vicinity of a band inversion due to their ferroelectric properties}

A.E. Klimov ${ }^{1,2}$, A.N. Akimov 1 , I.O. Akhundov ${ }^{1}$, V.A. Golyashov ${ }^{1,3}$, D.V. Gorshkov' ${ }^{1}$ D.V. Ishchenko ${ }^{1}$, E.V. Matyushenko ${ }^{1}$, I.G. Neizvestny ${ }^{1,2}$,

G.Yu. Sidorov ${ }^{1}$, S.P. Suprun ${ }^{1}$, A.S. Tarasov ${ }^{1}$, O.E. Tereshchenko ${ }^{1,3}$, V.S. Epov ${ }^{1}$

${ }^{1}$ Rzhanov Institute of Semiconductor Physics,

Russian Academy of Sciences,

630090 Novosibirsk, Russia

${ }^{2}$ Novosibirsk State Technical University,

630073 Novosibirsk, Russia

${ }^{3}$ Novosibirsk State University,

630090 Novosibirsk, Russia

Abstract The characteristics of MIS structures based on insulating PbSnTe:In films with compositions in the vicinity of a band inversion grown by molecular beam epitaxy (MBE) were studied. It has been shown that a number of their features can be caused by a ferroelectric phase transition with a Curie temperature in the range $T \approx 15-20 \mathrm{~K}$. 